\title{
La chantilly est mauvaise pour la santé
}

\section{Chantilly Is Bad for Health}

\author{
Y. Auffret $\cdot$ C. Petit $\cdot$ F. Lapostolle
}

Reçu le 23 mai 2018 ; accepté le 13 juillet 2018

(C) SFMU et Lavoisier SAS 2018

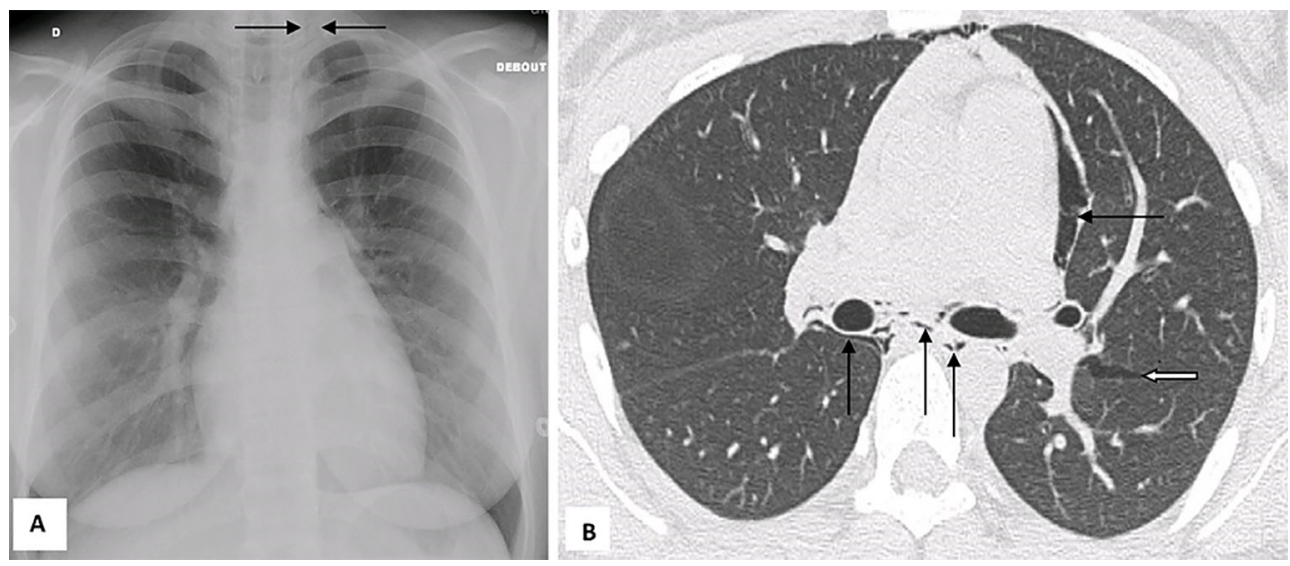

Fig. 1 Imagerie thoracique. A. Radio thoracique : emphysème cervical sous-cutané. Flèche noire : hyperclarté témoignant de l'emphysème sous-cutané. B. Scanner thoracique : pneumomédiastin. Flèche noire : hyperclarté délimitant les structures médiastinales. Flèche blanche : dissection de l'interstitium pulmonaire

Une femme de 20 ans sans antécédents particuliers, au cours d'une « soirée d'intégration », a aspiré du protoxyde d'azote (NO) sous pression par le biais d'un siphon, appareil permettant de servir la crème chantilly. Le lendemain, elle s'est plainte de douleurs du cou et de la partie supérieure du thorax associées à un emphysème sous-cutané du cou et des épaules. Elle s'est présentée aux urgences où la pression artérielle était de 120/63 $\mathrm{mmHg}$, la fréquence cardiaque : $56 /$ min, la saturation en oxygène en air ambiant : $100 \%$, la fréquence respiratoire : 12/min. L'examen clinique retrouvait l'emphysème sous-cutané. La radiographie thoracique mettait en évidence un emphysème cervical sous-cutané (signe de Minnigerode) (Fig. 1A). Un scanner thoracique a confirmé l'emphysème sous-cutané et mis en évidence un

Y. Auffret $(\triangle) \cdot$ C. Petit

Urgences, centre hospitalier de Quimper,

14 bis, avenue Yves-Thépot, F-29107 Quimper, France

e-mail : y.aunmail@yahoo.fr

\section{F. Lapostolle}

Samu 93, EA 3509, UFR Enseignement-Qualité,

hôpital Avicenne, université Paris-XIII, Sorbonne-Paris-Cité,

125, rue de Stalingrad, F-93009 Bobigny, France pneumomédiastin (Fig. 1B). La patiente a été hospitalisée pour prise en charge de la douleur et surveillance pendant 24 heures. L'évolution a été rapidement favorable. L'augmentation de la pression dans les voies respiratoires peut être responsable de pneumomédiastin. Cela a déjà été décrit chez les consommateurs de drogue qui utilisent la manœuvre de Valsalva et/ou le bouche-à-bouche pour augmenter la diffusion des drogues. Le NO est la star montante des soirées festives, notamment chez les étudiants, il est utilisé pour son effet euphorisant [1]. Son accès est facile dans les supermarchés (produits de cuisine : siphon à chantilly). À notre connaissance, seuls deux cas de pneumomédiastin chez l'homme ont été publiés après intoxication volontaire dans un but récréatif $[1,2]$.

\section{Références}

1. McDermott R, Tsang K, Hamilton N, et al (2015) Recreational nitrous oxide inhalation as a rare cause of spontaneous pneumomediastinum. BMJ Case Rep pii:bcr 209750

2. Lipuma JP, Wellman J, Stern HP (1982) Nitrous oxide abuse: a new cause of pneumomediastinum. Radiology 145:602 